\title{
Low Complexity Moving Target Parameter Estimation for MIMO Radar using 2D-FFT
}

\author{
Seifallah Jardak, Sajid Ahmed, Senior Member, IEEE, and Mohamed-Slim Alouini, Fellow, IEEE \\ Computer, Electrical, and Mathematical Science and Engineering (CEMSE) Division \\ King Abdullah University of Science and Technology (KAUST) \\ Thuwal, Makkah Province, Kingdom of Saudi Arabia \\ Email: \{seifallah.jardak, sajid.ahmed, slim.alouini\}@kaust.edu.sa
}

\begin{abstract}
In multiple-input multiple-output radar, to localize a target and estimate its reflection coefficient, a given cost function is usually optimized over a grid of points. The performance of such algorithms is directly affected by the grid resolution. Increasing the number of grid points enhances the resolution of the estimator but also increases its computational complexity exponentially. In this work, two reduced complexity algorithms are derived based on Capon and amplitude and phase estimation (APES) to estimate the reflection coefficient, angular location and, Doppler shift of multiple moving targets. By exploiting the structure of the terms, the cost-function is brought into a form that allows us to apply the two-dimensional fast-Fouriertransform (2D-FFT) and reduce the computational complexity of estimation. Using low resolution 2D-FFT, the proposed algorithm identifies sub-optimal estimates and feeds them as initial points to the derived Newton gradient algorithm. In contrast to the gridbased search algorithms, the proposed algorithm can optimally estimate on- and off-the-grid targets in very low computational complexity. A new APES cost-function with better estimation performance is also discussed. Generalized expressions of the Cramér-Rao lower bound are derived to asses the performance of the proposed algorithm.
\end{abstract}

Index Terms-MIMO-radar, Reflection coefficient, Doppler shift, Spatial location, two-dimensional fast-Fourier-transform, Cramér-Rao lower bound.

\section{INTRODUCTION}

$\mathbf{T}$ HE radar system that transmits independent or partially correlated waveforms and uses multiple antennas at the transmitter and the receiver is called a multiple-input multipleoutput (MIMO) radar. In MIMO radar, transmitting and receiving antennas can be collocated [1] or widely spaced [2]. In collocated MIMO radar, fully independent waveforms provide extra degrees-of-freedom (DOF), improved spatial resolution, and better parametric identifiability [3]-[5], at the price of reducing the transmit coherent processing gain. However, carefully designed correlated waveforms can electronically steer the beam in different directions of interest [1], [6], [7]. This increases the signal-to-noise-ratio (SNR) at the receiver and improves the estimation performance of the system. Alternatively, widely spaced MIMO radar provides spatial diversity and is more robust against the scintillation effect [8]. The focus of this paper is on the collocated MIMO radar.

This paper is an extended version of the work presented in the International Radar Conference, Lille, France, Oct. 2014. This work was funded by a CRG grant from the KAUST Office of Competitive Research Fund (OCRF).
Several adaptive techniques are developed to estimate the parameters of stationary targets in the collocated MIMO radar case. For example, in [9] and [10], a simple onedimensional search problem is introduced to jointly estimate the reflection coefficient and the direction-of-arrival of stationary targets in the monostatic MIMO radar case. In [11], a 2D-Capon algorithm is applied to estimate the directionof-departure and direction-of-arrival of multiple targets in the bistatic MIMO radar scenario. Besides, a more efficient target detection and parameter estimation algorithm based on the Parallel Factor (PARAFAC) analysis is introduced in [12]. However, when the targets are moving, the Doppler shift is an additional unknown parameter that should be estimated. In [13], the authors applied the estimation-of-signal-parametersvia-rotational-invariant-techniques (ESPRIT) and derived a reduced complexity algorithm for the joint estimation of the angle and Doppler frequencies. In [14], [15], authors have proposed a method based on time division multiplexing to estimate only the direction-of-arrival of moving targets.

In this paper, to estimate the reflection coefficient, spatial location, and Doppler shift of multiple moving targets, using collocated MIMO radar, our contributions are given below:

- The implementation of Capon and amplitude-and-phaseestimation (APES) using two-dimensional fast-Fouriertransform (2D-FFT) is introduced in [16]-[19]. However, as far as the authors know, it was never been used for the estimation of the spatial location and Doppler shift. We show that if the target is moving, the cost-function in its original form cannot be used to straightforwardly apply the 2D-FFT.

- By exploiting the structure of the vectors and matrices, we manipulate the terms of the cost-function and bring it in another form that allows us to apply the 2D-FFT to estimate the spatial location and Doppler shift. This reduces the computational complexity of the two-dimensional grid search problem from $O\left(N^{3}\right)$ to $O\left(N^{2} \log _{2}(N)\right)$, where $N$ is the number of grid points.

- The estimation of the target's reflection coefficient is written in closed-form and depends on the spatial location and Doppler shift. Contrarily to conventional algorithms [10], [20] where the estimate of the spatial location corresponds to the value where the reflection coefficient is maximized, we estimated the spatial location and 
Doppler shift by minimizing a new cost-function. This significantly improves the estimation performance.

- Low resolution estimates are used as initial points for the derived Newton gradient algorithm, which can yield onand off-the-grid estimates.

- To assess the estimator's performance, the generalized expressions of the Cramér-Rao lower bound (CRLB) are derived and compared with the mean-square-estimationerror (MSEE) of each parameter. The CRLB expressions are function of the transmitted signals and the covariance matrix of the colored Gaussian noise.

The organization of the paper is as follows. In the following section, the problem is formulated. The estimators of the different parameters are derived in Section III. In Section IV, the Fisher-information-matrix (FIM) and the CRLB of the parameters are derived. The derivations of the iterative Newton algorithm are detailed in Section V. Simulation results are presented in Section VI. Finally, conclusion is drawn in Section VII.

Notation: Bold upper case letters, $\mathbf{X}$, and lower case letters, $\mathbf{x}$, respectively denote matrices and vectors. The identity matrix of dimension $n \times n$ is denoted by $\mathbf{I}_{n}$ and $\mathbf{D}_{n}$ denotes the diagonal matrix whose elements are $\left[\begin{array}{llll}0 & 1 & \cdots & n-1\end{array}\right]$. Transpose, conjugate and conjugate transposition of a matrix are respectively denoted by $(\cdot)^{T},(\cdot)^{*}$ and $(\cdot)^{H}$, while the statistical expectation is denoted by $\mathbb{E}\{$.$\} . The real, imaginary,$ and absolute value of a complex variable are respectively represented by $(\cdot)^{\Re},(\cdot)^{\Im}$, and $|x|$. The subscript $t$ in $\beta_{t}$ refers to the true target parameter while $\beta$ and $\hat{\beta}$ refer respectively to the dummy variable and the final estimated variable. This notation applies also for $\omega, \bar{\omega}$, and $\mathbf{w}$.

\section{Problem Formulation}

Consider a narrowband MIMO radar system with uniformlinear-arrays (ULAs) at the transmitter and receiver. Let $d_{T}$ and $d_{R}$ respectively denote the inter-element spacing between the $n_{T}$ transmitting and $n_{R}$ receiving antennas and $\gamma$ be the ratio $d_{T} / d_{R}$. Let $\beta_{t}$ be the reflection coefficient of a generic target located at an angle $\theta_{t}$ and moving with a normalized angular Doppler shift $\omega_{t}$. The target's location $\theta_{t}$ can be considered as a normalized spatial angular frequency defined by $\bar{\omega}_{t}=2 \pi \frac{d_{R}}{\lambda} \sin \left(\theta_{t}\right)$ where $\lambda$ is the wavelength of the transmitted signals.

Let $\left\{x_{k, n}\right\}, k=0,1, \cdots, n_{T}-1, \quad n=0,1, \cdots, N-1$, denote the baseband signal transmitted from the $k^{\text {th }}$ antenna at time index $n$. The received signal $\left\{y_{m, n}\right\}$ can be expressed as below

$$
\begin{gathered}
y_{m, n}=\beta_{t} e^{j \omega_{t} n} e^{j \bar{\omega}_{t} m} \sum_{k=0}^{n_{T}-1} e^{j \gamma \bar{\omega}_{t} k} x_{k, n}+z_{m, n}\left(\omega_{t}, \bar{\omega}_{t}\right), \\
m=0,1, \cdots, n_{R}-1, n=0,1, \cdots, N-1,
\end{gathered}
$$

where $z_{m, n}\left(\omega_{t}, \bar{\omega}_{t}\right)$ denotes the residual term which includes the unmodelled noise, static clutter, and intentional or unintentional jamming.

In [20], [21], the authors studied the following model

$$
y_{m, n}=\alpha_{t}\left(\omega_{t}, \bar{\omega}_{t}\right) e^{j \omega_{t} n+\bar{\omega}_{t} m}+z_{m, n}\left(\omega_{t}, \bar{\omega}_{t}\right),
$$

where the variable of interest $\alpha_{t}(\omega, \bar{\omega})$ can be directly computed by applying the $2 \mathrm{D}-\mathrm{FFT}$ on the received signals. However, when comparing (1) with (2), it can be noticed that, because of the term $\sum_{k=0}^{n_{T}-1} e^{j \gamma \bar{\omega}_{t} k} x_{k, n}$, the 2D-FFT cannot be directly applied on the received signals to estimate the reflection coefficient $\beta_{t}$. Hence, the problem in (1) needs to be handled differently.

The transmit and receive steering vectors for a normalized spatial angular frequency $\bar{\omega}$ are defined respectively as follows

$$
\begin{aligned}
& \mathbf{a}_{T}(\bar{\omega})=\left[\begin{array}{llll}
1 & e^{j \gamma \bar{\omega}} & \cdots & e^{j \gamma \bar{\omega}\left(n_{T}-1\right)}
\end{array}\right]^{T}, \\
& \mathbf{a}_{R}(\bar{\omega})=\left[\begin{array}{llll}
1 & e^{j \bar{\omega}} & \cdots & e^{j \bar{\omega}\left(n_{R}-1\right)}
\end{array}\right]^{T} .
\end{aligned}
$$

Thus, the received signal can be written in the following vector form

$$
\mathbf{y}_{n}=\beta_{t} e^{j \omega_{t} n} \mathbf{a}_{R}\left(\bar{\omega}_{t}\right) \mathbf{a}_{T}^{T}\left(\bar{\omega}_{t}\right) \mathbf{x}_{n}+\mathbf{z}_{n}
$$

where

$$
\mathbf{y}_{n}=\left[\begin{array}{llll}
y_{0, n} & y_{1, n} & \cdots & y_{n_{R}-1, n}
\end{array}\right]^{T},
$$

is a column vector containing the received signals at time index $n$. Similar definition is applied to construct $\mathbf{x}_{n}$ and $\mathbf{z}_{n}$.

The problem is to estimate the target parameters, namely the reflection coefficient $\beta_{t}$, the Doppler angular frequency $\omega_{t}$ and the spatial angular frequency $\bar{\omega}_{t}$ using the received signals $\mathbf{y}_{n}$.

\section{Proposed Parameter Estimation}

Let $\mathbf{R}_{x}=\mathbb{E}\left\{\mathbf{x}_{n} \mathbf{x}_{n}^{H}\right\}$ be the covariance matrix of the transmitted waveforms, which can be fully independent or partially correlated. To enhance the estimation performance, the received signal is multiplied with a beamformer weight vector, w, after which the received signal can be written as

$$
\mathbf{w}^{H} \mathbf{y}_{n}=\beta_{t} e^{j \omega_{t} n} \mathbf{w}^{H} \mathbf{a}_{R}\left(\bar{\omega}_{t}\right) \mathbf{a}_{T}^{T}\left(\bar{\omega}_{t}\right) \mathbf{x}_{n}+\mathbf{w}^{H} \mathbf{z}_{n} .
$$

In this work, two adaptive beamforming methods are considered. First, the Capon beamformer [22], [23] is discussed. Next, the derivation is extended to the APES beamformer case [24], [25].

\section{A. Capon Estimator}

The Capon beamformer maximizes the signal-tointerference-plus-noise-ratio (SINR) and suppresses noise, interference and jamming while keeping the desired signal undistorted. If the covariance matrix of the received samples is denoted by $\mathbf{R}_{y}=\mathbb{E}\left\{\mathbf{y}_{n} \mathbf{y}_{n}^{H}\right\}$, the Capon beamformer is the solution of the following constrained optimization problem [9]

$$
\mathbf{w}_{c}=\underset{\mathbf{w}}{\operatorname{argmin}} \mathbf{w}^{H} \mathbf{R}_{y} \mathbf{w}, \text { subject to } \mathbf{w}^{H} \mathbf{a}_{R}(\bar{\omega})=1 .
$$

It should be noted that the covariance matrix $\mathbf{R}_{y}$ depends on the true parameters of the target. Solving (5) yields to the following expression of the Capon beamformer

$$
\mathbf{w}_{c}(\bar{\omega})=\frac{\mathbf{R}_{y}^{-1} \mathbf{a}_{R}(\bar{\omega})}{\mathbf{a}_{R}^{H}(\bar{\omega}) \mathbf{R}_{y}^{-1} \mathbf{a}_{R}(\bar{\omega})}
$$


The Capon estimate of $\beta_{t}$ is defined as the minimizer of the following cost-function

$$
\begin{aligned}
\hat{\beta}_{c}(\omega, \bar{\omega}) & =\underset{\beta}{\operatorname{argmin}} \mathbb{E}\left\{\left|\mathbf{w}_{c}^{H}(\bar{\omega}) \mathbf{y}_{n}-\beta e^{j \omega n} \mathbf{a}_{T}^{T}(\bar{\omega}) \mathbf{x}_{n}\right|^{2}\right\} \\
& =\frac{\mathbb{E}\left\{e^{-j \omega n} \mathbf{w}_{c}^{H}(\bar{\omega}) \mathbf{y}_{n} \mathbf{x}_{n}^{H} \mathbf{a}_{T}^{*}(\bar{\omega})\right\}}{p(\bar{\omega})} \\
& =\frac{\mathbb{E}\left\{e^{-j \omega n} \mathbf{a}_{R}^{H}(\bar{\omega}) \mathbf{R}_{y}^{-1} \mathbf{y}_{n} \mathbf{x}_{n}^{H} \mathbf{a}_{T}^{*}(\bar{\omega})\right\}}{p(\bar{\omega})\left(\mathbf{a}_{R}^{H}(\bar{\omega}) \mathbf{R}_{y}^{-1} \mathbf{a}_{R}(\bar{\omega})\right)}
\end{aligned}
$$

where

$$
p(\bar{\omega})=\mathbf{a}_{T}^{T}(\bar{\omega}) \mathbf{R}_{x} \mathbf{a}_{T}^{*}(\bar{\omega}),
$$

is the transmitted power at a normalized spatial angular frequency $\bar{\omega}$.

Evaluating the expression of $\hat{\beta}_{c}$ at each grid point $(\omega, \bar{\omega})$ requires high computational complexity. Thus, a more efficient implementation is derived. Let us define $\mathbf{r}_{n}=\mathbf{R}_{y}^{-1} \mathbf{y}_{n}$. So, the numerator of (7) can be written as

$$
\begin{aligned}
\mathbb{E} & \left\{e^{-j \omega n} \mathbf{a}_{R}^{H}(\bar{\omega}) \mathbf{r}_{n} \mathbf{x}_{n}^{H} \mathbf{a}_{T}^{*}(\bar{\omega})\right\} \\
& =\frac{1}{N} \sum_{n=0}^{N-1} e^{-j \omega n} \sum_{p=0}^{n_{T}-1} \sum_{q=0}^{n_{R}-1} r_{q, n} x_{p, n}^{*} e^{-j \bar{\omega}(\gamma p+q)} \\
& =\frac{1}{N} \sum_{n=0}^{N-1} \sum_{m=0}^{\gamma\left(n_{T}-1\right)}\left(\sum_{i=0}^{n_{R}-1} r_{m-i \gamma, n} x_{i, n}^{*}\right) e^{-j \bar{\omega} m} e^{-j \omega n} \\
& =\frac{1}{N} \sum_{n=0}^{N-1} \sum_{m=0}^{\gamma\left(n_{T}-1\right)} s_{m, n} e^{-j \bar{\omega} m} e^{-j \omega n},
\end{aligned}
$$

where $s_{m, n}=\sum_{i=0}^{n_{T}-1} r_{m-i \gamma, n} x_{i, n}^{*}$. Let $\mathbf{S}$ be a $\gamma\left(n_{T}-1\right)+$ $n_{R}-1 \times N$ complex matrix whose columns are

$$
\mathbf{s}_{n}=\left[\begin{array}{llll}
s_{0, n} & s_{1, n} & \cdots & s_{\gamma\left(n_{T}-1\right)}, n \\
+n_{R}-1
\end{array}\right]^{T}=\tilde{\mathbf{R}}_{n} \mathbf{x}_{n}^{*}
$$

and

$$
\tilde{\mathbf{R}}_{n}=\left[\begin{array}{cccc}
\mathbf{r}_{n} & \mathbf{0}_{\gamma \times 1} & \cdots & \mathbf{0}_{\gamma \times 1} \\
0 & \mathbf{r}_{n} & \ddots & \vdots \\
\vdots & \ddots & \ddots & \mathbf{0}_{\gamma \times 1} \\
0 & \cdots & 0 & \mathbf{r}_{n}
\end{array}\right] \in \mathcal{C}^{\gamma\left(n_{T}-1\right)} \times n_{R}-1
$$

Interestingly, the generation of new samples $s_{m, n}$ by multiplying $\mathbf{r}_{n}$ with $\mathbf{x}_{n}$ allows us to apply 2D-FFT. Therefore, by defining the matrix $\mathcal{S}=\frac{1}{N} 2 \mathrm{D}$-FFT $\left(\mathbf{S}^{T}\right)$, the target's spatial location and Doppler frequency can be jointly estimated as follows

$$
\begin{aligned}
\hat{\omega}_{c}, \hat{\bar{\omega}}_{c} & =\underset{\omega, \bar{\omega}}{\operatorname{argmax}}\left|\hat{\beta}_{c}(\omega, \bar{\omega})\right|^{2} \\
& =\underset{\omega, \bar{\omega}}{\operatorname{argmax}} \frac{|\mathcal{S}(\omega, \bar{\omega})|^{2}}{p(\bar{\omega})^{2}\left(\mathbf{a}_{R}^{H}(\bar{\omega}) \mathbf{R}_{y}^{-1} \mathbf{a}_{R}(\bar{\omega})\right)^{2}} .
\end{aligned}
$$

Hence, using the 2D-FFT approach, the number of operations required to compute the term in (9) is reduced from $\mathcal{O}\left(\mathcal{N}_{\omega} \times \mathcal{N}_{\bar{\omega}} \times N\right)$ to $\mathcal{O}\left(\mathcal{N}_{\omega} \times \mathcal{N}_{\bar{\omega}} \times \log _{2}\left(\mathcal{N}_{\omega} \times \mathcal{N}_{\bar{\omega}}\right)\right)$ where $\mathcal{N}_{\omega}$ and $\mathcal{N}_{\bar{\omega}}$ are respectively the number of grid points for $\omega$ and $\bar{\omega}$. It should be noted that more efficient algorithms [17], [18] are proposed to invert the received covariance matrix $\mathbf{R}_{y}$. However, because such approaches can be directly implemented in this problem, the authors did not expand that aspect.

The resolution of the 2D-FFT depends mainly on the number of samples. Hence, from (9), one concludes that using more time samples, i.e. increasing $\mathrm{N}$, enhances the Doppler resolution of the cost-function. However, to enhance the spatial resolution, one can increase the number of transmit and receive antennas or increase $\gamma$ which results in a longer virtual steering vector. The effect of using a longer virtual array is shown in Section VI.

\section{B. APES Estimator}

By following [24], the APES beamformer is formulated as

$$
\hat{\beta}_{a}, \mathbf{w}_{a}=\left\{\begin{array}{l}
\underset{\beta, \mathbf{w}}{\operatorname{argmin}} \mathbb{E}\left\{\left|\mathbf{w}^{H}(\bar{\omega}) \mathbf{y}_{n}-\beta e^{j \omega n} \mathbf{a}_{T}^{T}(\bar{\omega}) \mathbf{x}_{n}\right|^{2}\right\}, \\
\text { subject to } \mathbf{w}^{H} \mathbf{a}_{R}(\bar{\omega})=1 .
\end{array}\right.
$$

Again, by differentiating the above cost-function with respect to the complex conjugate $\beta^{*}$ and equating it to 0 , the expression of the minimizing reflection coefficient $\beta$ can be written as

$$
\beta=\frac{\mathbf{w}^{H}(\bar{\omega}) \tilde{\mathbf{v}}(\omega, \bar{\omega})}{p(\bar{\omega})},
$$

where $\tilde{\mathbf{v}}(\omega, \bar{\omega})=\mathbb{E}\left\{e^{-j \omega n} \mathbf{y}_{n} \mathbf{x}_{n}^{H} \mathbf{a}_{T}^{*}(\bar{\omega})\right\}$. By substituting (14) in (13), the APES beamformer is determined as

$$
\mathbf{w}_{a}=\frac{\mathbf{Q}^{-1}(\omega, \bar{\omega}) \mathbf{a}_{R}(\bar{\omega})}{\mathbf{a}_{R}^{H}(\bar{\omega}) \mathbf{Q}^{-1}(\omega, \bar{\omega}) \mathbf{a}_{R}(\bar{\omega})}
$$

where

$$
\mathbf{Q}(\omega, \bar{\omega})=\mathbf{R}_{y}-\frac{\tilde{\mathbf{v}}(\omega, \bar{\omega}) \tilde{\mathbf{v}}^{H}(\omega, \bar{\omega})}{p(\bar{\omega})} .
$$

(For notational convenience, we sometimes drop the dependence on $\omega$ and $\bar{\omega}$ below.)

Usually [20], [21], the APES estimates of $\omega_{t}$ and $\bar{\omega}_{t}$ are defined as the maximizers of the below cost-function

$$
\begin{aligned}
\hat{\omega}_{a}, \hat{\bar{\omega}}_{a} & =\underset{\omega, \bar{\omega}}{\operatorname{argmax}}\left|\hat{\beta}_{a}(\omega, \bar{\omega})\right|^{2} \\
& =\underset{\omega, \bar{\omega}}{\operatorname{argmax}} \frac{\mathbf{w}_{a}^{H}(\bar{\omega}) \tilde{\mathbf{v}}(\omega, \bar{\omega}) \tilde{\mathbf{v}}^{H}(\omega, \bar{\omega}) \mathbf{w}_{a}^{H}(\bar{\omega})}{p(\bar{\omega})^{2}} \\
& =\underset{\omega, \bar{\omega}}{\operatorname{argmax}} \frac{\mathbf{a}_{R}^{H}(\bar{\omega}) \mathbf{Q}^{-1} \tilde{\mathbf{v}} \tilde{\mathbf{v}}^{H} \mathbf{Q}^{-1} \mathbf{a}_{R}(\bar{\omega})}{p(\bar{\omega})^{2}\left(\mathbf{a}_{R}(\bar{\omega}) \mathbf{Q}^{-1} \mathbf{a}_{R}(\bar{\omega})\right)^{2}} .
\end{aligned}
$$

Using the Sherman-Morrison formula, the inverse of $\mathbf{Q}(\omega, \bar{\omega})$ is

$$
\mathbf{Q}^{-1}(\omega, \bar{\omega})=\mathbf{R}_{y}^{-1}+\frac{\mathbf{R}_{y}^{-1} \tilde{\mathbf{v}}(\omega, \bar{\omega}) \tilde{\mathbf{v}}^{H}(\omega, \bar{\omega}) \mathbf{R}_{y}^{-1}}{p(\bar{\omega})-\tilde{\mathbf{v}}^{H}(\omega, \bar{\omega}) \mathbf{R}_{y}^{-1} \tilde{\mathbf{v}}(\omega, \bar{\omega})} .
$$

Hence, (17) becomes equivalent to maximizing

$$
\left|\hat{\beta}_{a}\right|^{2}=\frac{\left|\mathbf{a}_{R}^{H} \mathbf{R}_{y}^{-1} \tilde{\mathbf{v}}\right|^{2}}{\left(\left|\mathbf{a}_{R}^{H} \mathbf{R}_{y}^{-1} \tilde{\mathbf{v}}\right|^{2}+\left(\mathbf{a}_{R}^{H} \mathbf{R}_{y}^{-1} \mathbf{a}_{R}\right)\left(p-\tilde{\mathbf{v}}^{H} \mathbf{R}_{y}^{-1} \tilde{\mathbf{v}}\right)\right)^{2}} .
$$


However, when minimizing the following cost-function

$$
\begin{aligned}
\hat{\omega}_{a}, \hat{\bar{\omega}}_{a} & =\underset{\omega, \bar{\omega}}{\operatorname{argmin}} \mathbb{E}\left\{\left|\mathbf{w}_{a}^{H}(\bar{\omega}) \mathbf{y}_{n}-\hat{\beta}_{a} e^{j \omega n} \mathbf{a}_{T}^{T}(\bar{\omega}) \mathbf{x}_{n}\right|^{2}\right\} \\
& =\underset{\omega, \bar{\omega}}{\operatorname{argmin}} \mathbf{w}_{a}^{H}(\bar{\omega}) \mathbf{Q} \mathbf{w}_{a}(\bar{\omega}) \\
& =\underset{\omega, \bar{\omega}}{\operatorname{argmax}} \mathbf{a}_{R}^{H}(\bar{\omega}) \mathbf{Q}^{-1} \mathbf{a}_{R}(\bar{\omega}) \\
& =\underset{\omega, \bar{\omega}}{\operatorname{argmax}} \mathbf{a}_{R}^{H} \mathbf{R}_{y}^{-1} \mathbf{a}_{R}+\frac{\left|\mathbf{a}_{R}^{H} \mathbf{R}_{y}^{-1} \tilde{\mathbf{v}}\right|^{2}}{p-\tilde{\mathbf{v}}^{H} \mathbf{R}_{y}^{-1} \tilde{\mathbf{v}}},
\end{aligned}
$$

a simpler expression is found and the simulation results show that it also has a better estimation performance.

The expression of the second term's numerator is equal to the absolute square of the expression in (9). Hence, the same 2D-FFT approach is again used to evaluate it. Now, a marginally different approach is proposed to compute the term $\tilde{\mathbf{v}}^{H} \mathbf{R}_{y}^{-1} \tilde{\mathbf{v}}$ in the denominator. First, the Cholesky decomposition [26] is used to design an upper triangular matrix $\mathbf{U}$ such that $\mathbf{U}^{H} \mathbf{U}=\mathbf{R}_{y}^{-1}$. Next, by defining the two column vectors $\tilde{\mathbf{r}}_{n}=\mathbf{U y}_{n}$ and $\mathbf{v}(\omega, \bar{\omega})=\mathbf{U} \tilde{\mathbf{v}}(\omega, \bar{\omega})=\mathbb{E}\left\{e^{-j \omega n} \tilde{\mathbf{r}}_{n} \mathbf{x}_{n}^{H} \mathbf{a}_{T}^{*}(\bar{\omega})\right\}$, we can write

$$
\begin{aligned}
\mathbf{v}_{i}(\omega, \bar{\omega}) & =\frac{1}{N} \sum_{n=0}^{N-1} \sum_{m=0}^{n_{T}-1} \tilde{r}_{i, n} x_{m, n}^{*} e^{-j \omega n} e^{-j \gamma \bar{\omega} m} \\
& =\frac{1}{N} \sum_{n=0}^{N-1} \sum_{m=0}^{\gamma\left(n_{T}-1\right)} \tilde{r}_{i, n} \bar{x}_{m, n}^{*} e^{-j \omega n} e^{-j \bar{\omega} m} \\
& =\frac{1}{N} \sum_{n=0}^{N-1} \sum_{m=0}^{\gamma\left(n_{T}-1\right)} \mathbf{V}_{i, m, n} e^{-j \omega n} e^{-j \bar{\omega} m}
\end{aligned}
$$

where $\mathbf{V}_{i, m, n}=\tilde{r}_{i, n} \bar{x}_{m, n}^{*}$ is a term of a three dimentional matrix $\mathbf{V}$ of size $n_{R} \times \gamma\left(n_{T}-1\right) \times N$ and $\bar{x}_{m, n}$ is an upsampled version of $x_{m, n}$ such that

$$
\begin{cases}\bar{x}_{\gamma m, n}=x_{m, n} & \text { for } m=0,1, \cdots, n_{T}-1, \\ \bar{x}_{m, n}=0 & \text { otherwise. }\end{cases}
$$

By defining the matrix $\mathcal{V}_{i}=\frac{1}{N} 2 \operatorname{D-FFT}\left(\mathbf{V}_{i}^{T}\right), i=$ $0, \cdots, n_{R}-1$, we can finally write

$$
\tilde{\mathbf{v}}^{H}(\omega, \bar{\omega}) \mathbf{R}_{y}^{-1} \tilde{\mathbf{v}}(\omega, \bar{\omega})=\sum_{i=0}^{n_{R}-1}\left|\mathcal{V}_{i}(\omega, \bar{\omega})\right|^{2} .
$$

Therefore, the cost-function in (20) can be reformulated as shown below

$$
\hat{\omega}_{a}, \hat{\bar{\omega}}_{a}=\underset{\omega, \bar{\omega}}{\operatorname{argmax}} \mathbf{a}_{R}^{H}(\bar{\omega}) \mathbf{R}_{y}^{-1} \mathbf{a}_{R}(\bar{\omega})+\frac{|\mathcal{S}(\omega, \bar{\omega})|^{2}}{p(\bar{\omega})-\|\mathcal{V}(\omega, \bar{\omega})\|_{2}^{2}} .
$$

Again, the 2D-FFT approach reduced the number of operations required to compute the term in (21) from $\mathcal{O}\left(n_{R} \times\right.$ $\left.\mathcal{N}_{\omega} \times \mathcal{N}_{\bar{\omega}} \times N\right)$ to $\mathcal{O}\left(n_{R} \times \mathcal{N}_{\omega} \times \mathcal{N}_{\bar{\omega}} \times \log _{2}\left(\mathcal{N}_{\omega} \times \mathcal{N}_{\bar{\omega}}\right)\right)$. Similarly, it should be noticed that by increasing $\gamma$, the size of $\mathbf{V}$ increases. Hence, the 2D-FFT result is interpolated leading to higher spatial resolution.

To assess the performance of the presented estimators, the CRLB of all three parameters will be derived in the following section.

\section{CRAmÉR-RAo Lower Bound}

In this section, the CRLB is derived in the case where one moving target is present. The problem in (3) can be reformulated in a vector form as below

$$
\mathbf{y}=\mathbf{u}+\mathbf{z}
$$

where $\mathbf{y}=\left[\begin{array}{llll}\mathbf{y}_{n}^{T} & \mathbf{y}_{n+1}^{T} & \cdots & \mathbf{y}_{n+N-1}^{T}\end{array}\right]^{T}$ is a column vector that contains all received samples from time $n$ to $(n+N-$ 1). Also, $\mathbf{u}$ and $\mathbf{z}$ are defined similarly. Assuming that there are no interferers and that the noise samples follow a white Gaussian distribution with covariance matrix $\mathbf{R}_{z}$ independent of the target parameters, the FIM for the estimation of the vector of unknown parameters $\boldsymbol{\eta}=\left[\begin{array}{llll}\beta_{t}^{\Re} & \beta_{t}^{\Im} & \omega_{t} & \bar{\omega}_{t}\end{array}\right]$ can be found using the Slepian-Bangs formula [27]

$$
\begin{aligned}
\mathbf{F}(\boldsymbol{\eta}) & =2\left(\frac{\partial \mathbf{u}^{H}}{\partial \boldsymbol{\eta}}\left[\begin{array}{ccc}
\mathbf{R}_{z} & & 0 \\
& \ddots & \\
0 & & \mathbf{R}_{z}
\end{array}\right]^{-1} \frac{\partial \mathbf{u}}{\partial \boldsymbol{\eta}^{T}}\right)^{\Re} \\
& =2 \sum_{n=0}^{N-1}\left(\frac{\partial \mathbf{u}_{n}^{H}}{\partial \boldsymbol{\eta}} \mathbf{R}_{z}^{-1} \frac{\partial \mathbf{u}_{n}}{\partial \boldsymbol{\eta}^{T}}\right)^{\Re} .
\end{aligned}
$$

As detailed in Appendix A, the final expressions of the CLRB for the different parameters are

$$
\begin{aligned}
& \operatorname{CRLB}\left(\beta_{t}^{\Re}\right)=\frac{1}{2 N q p}\left(1+\frac{\Delta_{1} \beta_{t}^{\Im^{2}}+\Delta_{2} \beta_{t}^{\Im} \beta_{t}^{\Re}+\Delta_{3} \beta_{t}^{\Re 2}}{\alpha}\right), \\
& \operatorname{CRLB}\left(\beta_{t}^{\Im}\right)=\frac{1}{2 N q p}\left(1+\frac{\Delta_{1} \beta_{t}^{\Re^{2}}-\Delta_{2} \beta_{t}^{\Im} \beta_{t}^{\Re}+\Delta_{3} \beta_{t}^{\Im^{2}}}{\alpha}\right), \\
& \operatorname{CRLB}\left(\omega_{t}\right)=\frac{1}{2 N q p} \frac{q_{2}-\left|q_{1}\right|^{2}+\gamma^{2}\left(p_{02}-\left|p_{01}\right|^{2}\right)}{\alpha}, \\
& \operatorname{CRLB}\left(\bar{\omega}_{t}\right)=\frac{1}{2 N q p} \frac{p_{20}-p_{10}^{2}}{\alpha},
\end{aligned}
$$

where

$$
\begin{aligned}
\alpha=\mid & \left.\beta_{t}\right|^{2}\left(p_{20}-p_{10}^{2}\right)\left(q_{2}-\left|q_{1}\right|^{2}+\gamma^{2}\left(p_{02}-\left|p_{01}\right|^{2}\right)\right) \\
- & \left|\beta_{t}\right|^{2} \gamma^{2}\left(p_{11}^{\Re}-p_{01}^{\Re} p_{10}\right)^{2}, \\
\Delta_{1}= & p_{10}^{2}\left(q_{2}-\left|q_{1}\right|^{2}+\gamma^{2}\left(p_{02}-\left|p_{01}\right|^{2}\right)\right) \\
& +2 \gamma p_{10}\left(p_{10} p_{01}^{\Re}-p_{11}^{\Re}\right)\left(q_{1}^{\Re}+\gamma p_{01}^{\Re}\right) \\
& +\left(p_{20}-p_{10}^{2}\right)\left(q_{1}^{\Re}+\gamma p_{01}^{\Re}\right)^{2}, \\
\Delta_{2}= & 2\left(q_{1}^{\Im}+\gamma p_{01}^{\Im}\right)\left(\gamma p_{20} p_{01}^{\Re}-\gamma p_{10} p_{11}^{\Re}+q_{1}^{\Re}\left(p_{20}-p_{10}^{2}\right)\right), \\
\Delta_{3}= & \left(p_{20}-p_{10}^{2}\right)\left(q_{1}^{\Im}+\gamma p_{01}^{\Im}\right)^{2} .
\end{aligned}
$$

It should be noted that the expressions of the CRLB are independent of the angular Doppler shift $\omega$.

To find a more accurate estimator $\hat{\boldsymbol{\eta}}$, the solution of (12) and (24) should be determined with very high resolution. Thus, a large number of FFT points should be used to determine the estimates $\hat{\omega}_{t}$ and $\hat{\bar{\omega}}_{t}$ as presented in [28]. To reduce the computational cost and avoid using large number of FFT points, an iterative method is presented in the next section.

\section{ITERATIVE METHOD}

This method will use low resolution estimates $\hat{\omega}_{t}$ and $\hat{\bar{\omega}}_{t}$ as initial values to initialize the Newton algorithm and optimize 
the appropriate Capon and APES cost-functions rewritten below

$$
\begin{aligned}
\hat{\omega}_{c}, \hat{\bar{\omega}}_{c}=\underset{\omega, \bar{\omega}}{\operatorname{argmax}} \frac{|\mathcal{S}(\omega, \bar{\omega})|^{2}}{p(\bar{\omega})^{2}\left(\mathbf{a}_{R}^{H}(\bar{\omega}) \mathbf{R}_{y}^{-1} \mathbf{a}_{R}(\bar{\omega})\right)^{2}}, \\
\hat{\omega}_{a}, \hat{\bar{\omega}}_{a}=\underset{\omega, \bar{\omega}}{\operatorname{argmax}} \mathbf{a}_{R}^{H}(\bar{\omega}) \mathbf{R}_{y}^{-1} \mathbf{a}_{R}(\bar{\omega})+\frac{|\mathcal{S}(\omega, \bar{\omega})|^{2}}{p(\bar{\omega})-\|\mathcal{V}(\omega, \bar{\omega})\|_{2}^{2}} .
\end{aligned}
$$

Hence, the first and second order derivatives with respect to $\omega$ and $\bar{\omega}$ of the expressions $|\mathcal{S}(\omega, \bar{\omega})|^{2},\|\mathcal{V}(\omega, \bar{\omega})\|_{2}^{2}$, $\left(\mathbf{a}_{R}^{H}(\bar{\omega}) \mathbf{R}_{y}^{-1} \mathbf{a}_{R}(\bar{\omega})\right)$ and $p(\bar{\omega})$ are detailed in Appendix B. Compared to the steepest descent algorithm, the Newton iteration is more complex because it requires the evaluation and inversion of the Hessian. Fortunately, in our case, the Hessian is only a 2 by 2 matrix. Thus, the computational complexity of evaluating and inverting it is minor compared to the gain achieved by the reduced number of iteration. Thus, the overall computational cost of the Newton method is much lower than the Steepest descent method. We summarize the Newton optimization algorithm as follows:

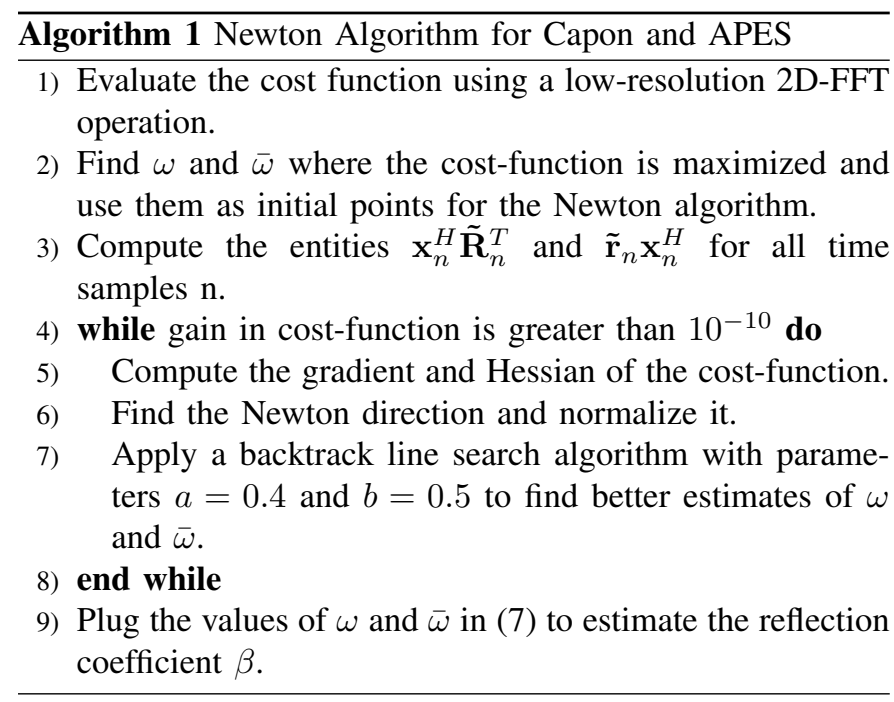

\section{Simulation Results}

This section presents the simulation results and demonstrates the performance of the proposed estimators. In all simulations, 10 transmit and 10 receive antennas are used and the inter-element spacing between antennas at the receiver is set to be half the wavelength, i.e. $d_{R}=\frac{\lambda}{2}$. The noise terms $\mathbf{z}_{n}$ are drawn from a zero mean spatially colored Gaussian distribution with an unknown covariance matrix. The $(p, q)$ th element of the unkown noise covariance matrix is defined as $\frac{1}{\mathrm{SNR}} 0.9^{|p-q|} e^{j \frac{\pi}{2}(p-q)}$.

In the first subsection, multiple targets are considered to investigate the effect of the beampattern design on the performance of our algorithms. In the second subsection, the performance of our estimators will be assessed by comparing their MSEE with the derived CRLB under different scenarios. The third subsection illustrates the computational gain achieved by the iterative algorithm.

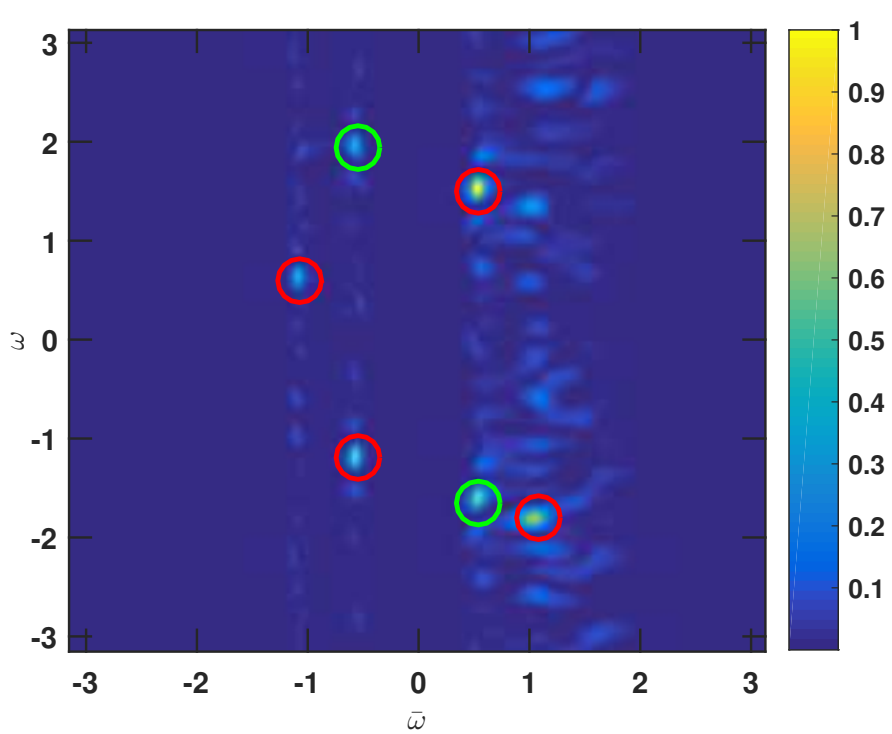

(a) Capon

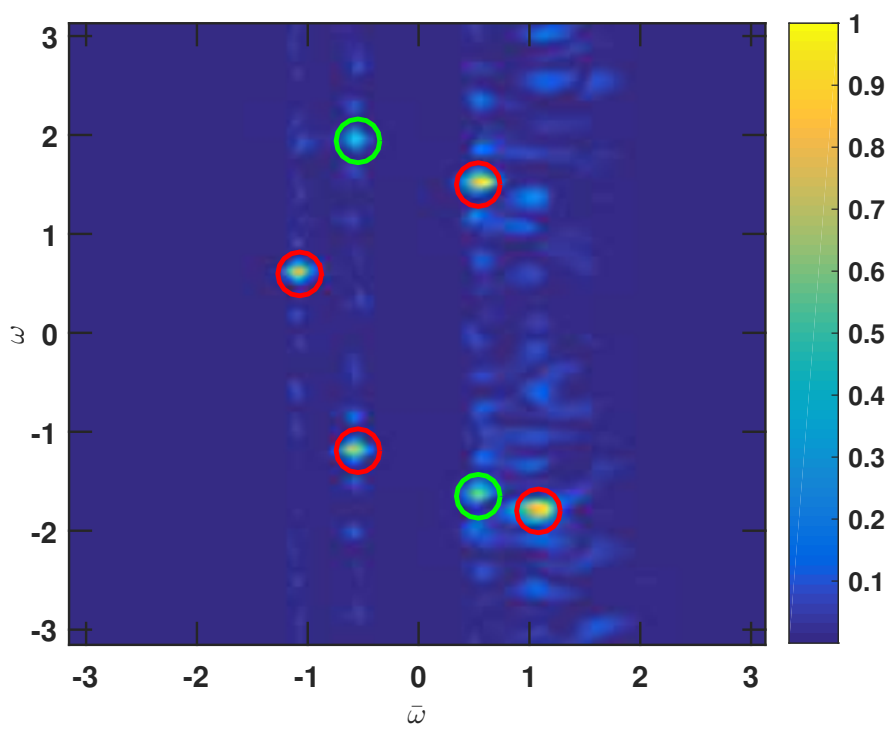

(b) APES

Fig. 1. Normalized amplitude of the different cost-functions derived for the (a) Capon and (b) APES algorithms as function of $\omega$ and $\bar{\omega}$ at $\mathrm{SNR}=0 \mathrm{~dB}$. The red circles indicate the location of targets and the green circles indicate false peaks (cost-function higher than 0.5). Here, the transmitted signals are fully independent.

\section{A. Effect of the beampattern design on the detection perfor- mance:}

Setting SNR $=0 \mathrm{~dB}, \mathrm{~N}=32$ snapshots are transmitted to detect 4 targets with parameters given in Table I. The Capon (12) and APES (19) cost-functions are computed using 256 point 2D-FFT. The performance of fully independent waveforms is presented in Fig. 1. When comparing the plots (a) and (b) in Fig. 1, the cost-functions are indeed maximized around the target positions indicated by the red circles. However, as we are operating at low SNR, both plots present two more peaks marked by green circles which do not correspond to actual targets.

To remediate this issue without increasing the transmitted power, a correlated 8-PAM waveform is generated using the 


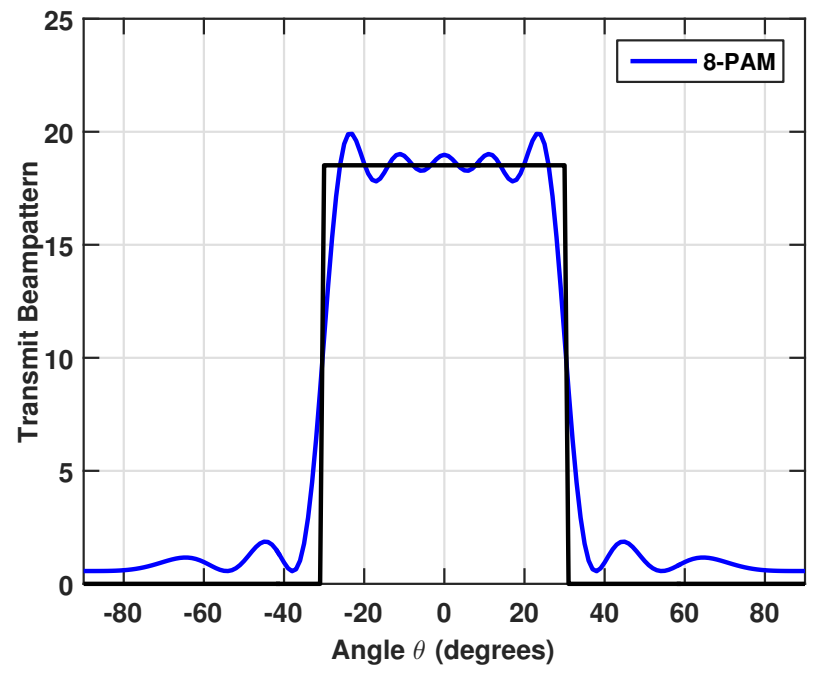

Fig. 2. The transmitted beampattern achieved by the designed 8-PAM signals. The region of interest is $\left[-30^{\circ}, 30^{\circ}\right]$.

TABLE I

The Simulation Parameters of the Multiple Targets

\begin{tabular}{|c||r|r|r|c|}
\hline & \multicolumn{1}{|c|}{$\omega_{t}$} & \multicolumn{1}{c|}{$\theta_{t}$} & \multicolumn{1}{c|}{$\bar{\omega}_{t}$} & $\beta_{t}$ \\
\hline \hline Target 1 & -1.2 & $-10^{\circ}$ & -0.55 & $(-2+j) / \sqrt{5}$ \\
\hline Target 2 & 1.5 & $10^{\circ}$ & 0.55 & $(-2+j) / \sqrt{5}$ \\
\hline Target 3 & 0.6 & $-20^{\circ}$ & -1.07 & $(-1+2 j) / \sqrt{5}$ \\
\hline Target 4 & -1.8 & $20^{\circ}$ & 1.07 & $(-2-j) / \sqrt{5}$ \\
\hline
\end{tabular}

beampattern design algorithm proposed in [7]. The designed signals maximize the transmitted power in the region of interest between $-30^{\circ}$ and $30^{\circ}$. Their transmitted power is illustrated in Fig. 2. The performance of the Capon and APES beamformers using the correlated signals is presented in Fig 3. It can be seen that when carefully designed correlated signals are used, the SNR is increased in the region of interest and the two false peaks are suppressed. In Fig. 1 and Fig. 3, it should be noted that the peaks of the Capon cost-function are narrower in the spatial direction $\bar{\omega}$. However, in the Doppler direction $\omega$, the peaks of the two beamformers have approximately the same resolution.

\section{B. Estimation performance:}

In the next simulations, one target of interest is located at $\theta_{t}=-5^{\circ}$ and has a reflection coefficient $\beta_{t}=\frac{-1+2 j}{\sqrt{5}}$. Two static scatterers are present at $-30^{\circ}$ and $50^{\circ}$ with interferenceto-noise ratio INR $=0 \mathrm{~dB}$. Here, we used $N=128$ snapshots of fully independent waveforms and the results are averaged over 1, 000 realizations. Since the CRLB of the parameters is independent of the target's Doppler frequency, $\omega_{t}$ is generated at each realization from a Gaussian random variable with mean $\pi / 2$ and variance 0.001 .

Because the two scatterers act as static targets, the costfunctions (12), (19) and, (24) are also maximized around their spatial location. Therefore, maximums with Doppler frequencies around 0 are neglected and we look for the first

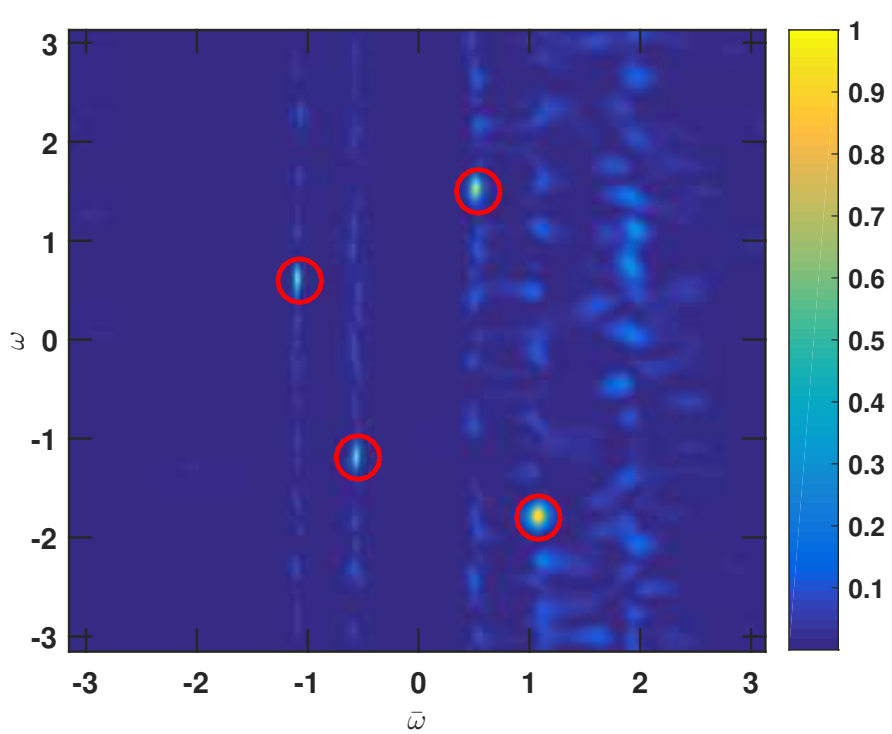

(a) Capon

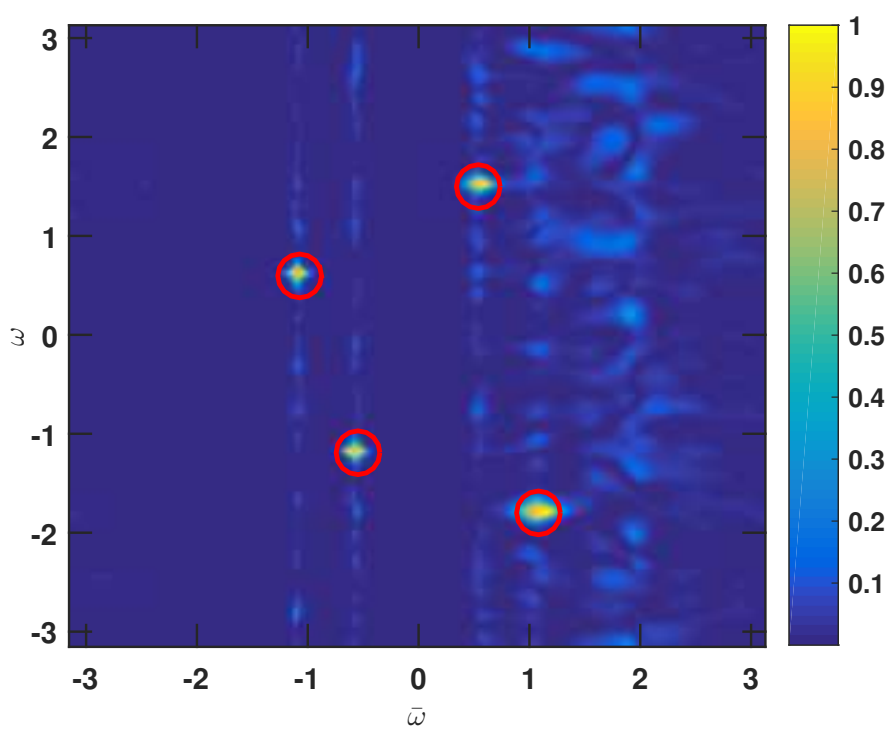

(b) APES

Fig. 3. Normalized amplitude of the different cost-functions derived for the (a) Capon and (b) APES algorithms as function of $\omega$ and $\bar{\omega}$ at $\mathrm{SNR}=0 \mathrm{~dB}$. The red circles indicate the location of targets. Here, the transmitted signals are designed to maximize the transmitted power between $-30^{\circ}$ and $30^{\circ}$.

maximum with non-null Doppler shift. Figure 4 presents the performance of the Capon estimator when half-wavelength inter-element spacing is used at the transmitter, i.e. $\gamma=1$. It can be seen that the MSEE of the spatial location estimator follows closely its CRLB with a $3 \mathrm{~dB}$ gap. However, the MSEE of the Doppler shift does not meet its CRLB. In fact, for the above simulation parameters, the Doppler MSEE is bounded by $-66 \mathrm{~dB}$.

Figure 5 presents the performance of the APES algorithm when maximizing the APES cost-function in (19). Although the APES algorithm is expected to outperform the Capon algorithm, it can be seen that the MSEE of the estimators are far from their respective CRLB.

However, when maximizing the cost-function (24), Fig. 6 shows a better performance. It can be seen that the MSEE 


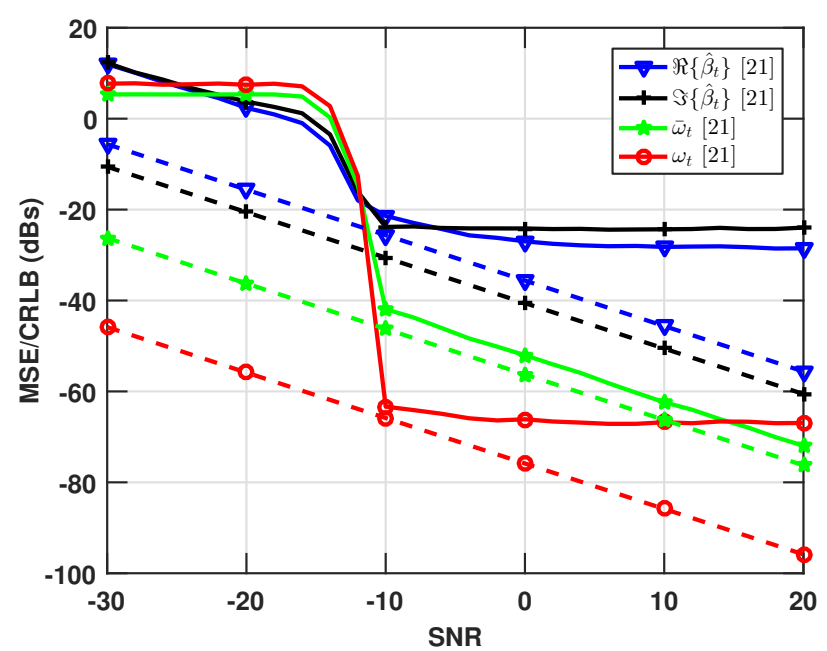

Fig. 4. Comparison of the CRLB (dashed lines) with the MSEE (solid lines) of the Capon algorithm in the estimation of $\beta_{t}, \omega_{t}$, and $\bar{\omega}_{t}$ using 2D-FFT with 1024 points $\gamma=1$.

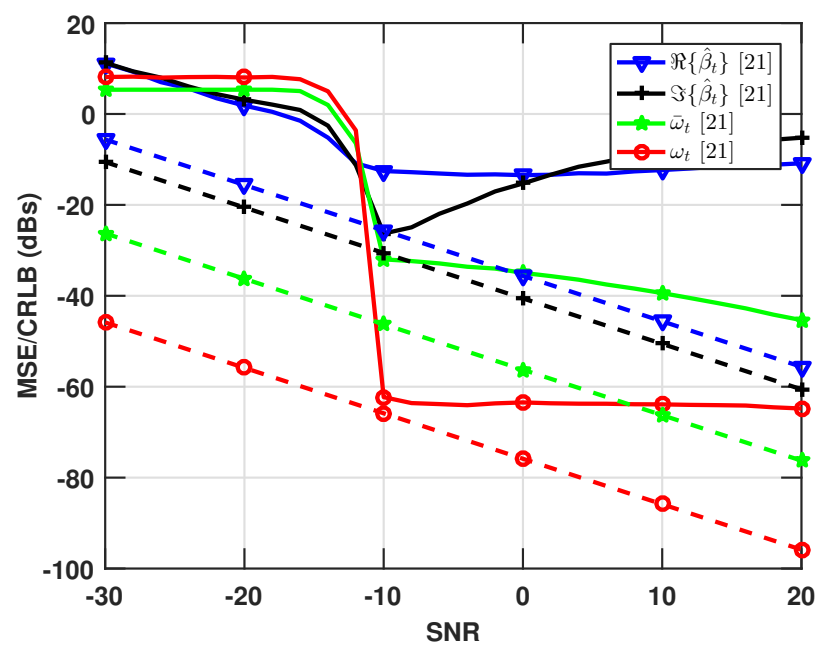

Fig. 5. Comparison of the CRLB (dashed lines) with the MSEE (solid lines) of the APES algorithm in the estimation of $\beta_{t}, \omega_{t}$, and $\bar{\omega}_{t}$ using cost-function (19), 2D-FFT with 1024 points and, $\gamma=1$.

of both spatial location and Doppler shift frequency follow their respective CRLB graphs. Moreover, when a longer virtual array [29]-[32] is constructed by setting $\gamma=2$, Fig. 7 shows how the MSEE of spatial location gets even closer to its CRLB. In fact, increasing the length of the virtual array improves mainly the spatial resolution. Thus, the CRLB of the Doppler shift remains almost unchanged while the CRLB of the spatial location expectedly decreases by $4 \mathrm{~dB}$.

When the SNR increases, the peaks of the Capon and APES cost-functions become narrower. Likewise, increasing the number of samples $\mathrm{N}$ or the number of transmit and receive antenna reduces the peak width of the cost-function. So, to avoid missing the maximum at high SNR values, we used a high resolution 2D-FFT with 1024 points. However, it should be noted that for SNR values less than $0 \mathrm{~dB}$, a 256 2D-FFT operation is adequate to detect the correct maximum.

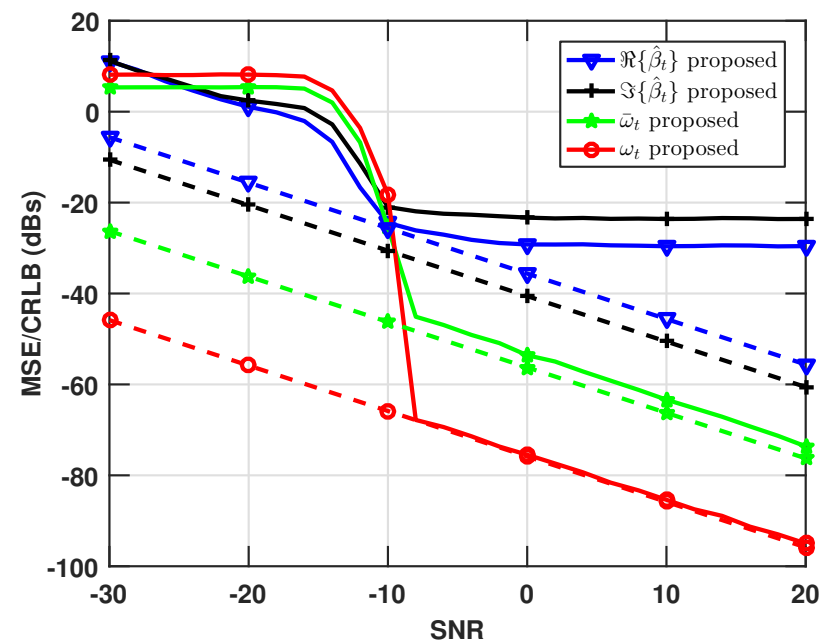

Fig. 6. Comparison of the CRLB (dashed lines) with the MSEE (solid lines) of the APES algorithm in the estimation of $\beta_{t}, \omega_{t}$, and $\bar{\omega}_{t}$ using cost-function (24), 2D-FFT with 1024 points and, $\gamma=1$.

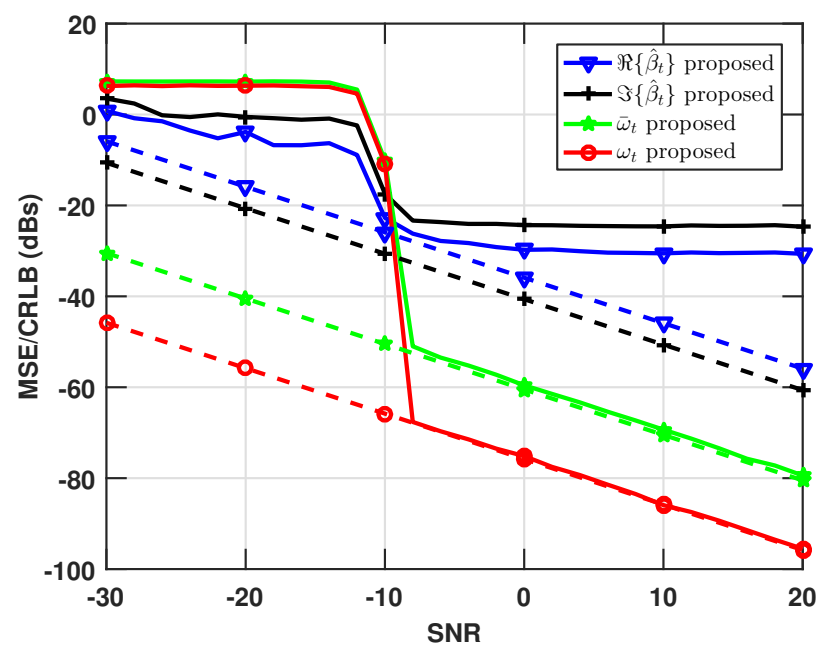

Fig. 7. Comparison of the CRLB (dashed lines) with the MSEE (solid lines) of the APES algorithm in the estimation of $\beta_{t}, \omega_{t}$, and $\bar{\omega}_{t}$ using cost-function (24), 2D-FFT with 1024 points and, $\gamma=2$.

\section{Computational performance:}

The gain in estimation performance achieved by APES compared to the Capon algorithm comes with a cost in computational complexity. As discussed in Section III, the Capon algorithm requires a single 2D-FFT operation while $n_{R}$ additional 2D-FFT operations are needed to compute the denominator of the APES cost-function.

The goal of this subsection is to compare the computational time required by two methods to converge to the maximum of the Capon and APES cost-functions. The first method is the Newton algorithm derived in Section V. The second one is a multiple 2D-FFT based approach. It consists in applying a local 2D-FFT in a much narrower region around the low resolution target parameter estimates. Due to this, the resolution of FFT increases, and we get estimates closer to the actual values of the parameters. This step is repeated around the new updated estimates in the further narrower region. To guarantee a fair comparison, the stopping criteria ensures that 


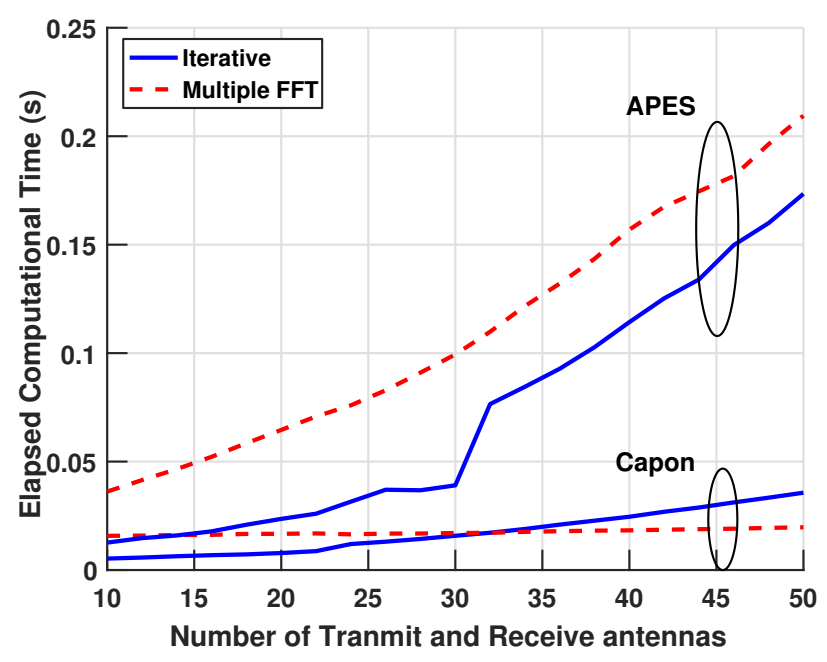

Fig. 8. Comparison of the average computational time needed to converge to the maximum of the Capon and APES cost-functions using the Newton iterative algorithm (solid lines) or the multiple 64 2D-FFT approach (dashed lines) under $\mathrm{SNR}=0 \mathrm{~dB}$.

the gain in cost-function is less than $10^{-10}$. We also verify that the estimators computed using both approaches achieve on average the same MSEE.

The computational time needed to converge to the maximum of the Capon and APES cost-functions is plotted in Fig 8 . It represents the elapsed time required to evaluate the steps from 3 to 8 in Algorithm 1. It can be seen that both methods converge to the maximum of the Capon cost-function faster than the APES. Moreover, the time difference increases as the number of antenna grows. Besides, in the case of Capon, Fig. 8 shows that the iterative algorithm converges faster than the multiple 64 point 2D-FFT when the number of transmit and receive antenna is less than 30 , i.e. $n_{R} \leq 30$ and $n_{T} \leq$ 30. Meanwhile, when maximizing the APES cost-function, the iterative algorithm converges faster than the multiple 2D-FFT method and achieves a gain of $40 \mathrm{~ms}$ on average. In Fig. 9, the average number of iteration is plotted. Interestingly, the number of iterations needed does not exceed 6 for the iterative algorithm and 3 for the multiple 2D-FFT approach. It should be noted that at each iteration, the iterative algorithm carries out a backtrack line search [33] with parameters $a=0.4$ and $b=0.5$. Yet, in total, the Newton algorithm converges faster than the multiple 2D-FFT approach.

\section{CONCLUSION}

In this work, a low complexity algorithm based on the 2DFFT is derived to estimate the reflection coefficient, spatial location and, Doppler shift of multiple moving targets using the Capon and APES beamformers. We also derived the CRLB of these parameters and showed that the Capon beamformer provides good spatial estimates. Besides, the APES beamformer provides better Doppler estimates at a greater computational complexity. We also studied the computational time required by the Newton algorithm and showed that the iterative method converges faster than the multiple 2D-FFT approach.

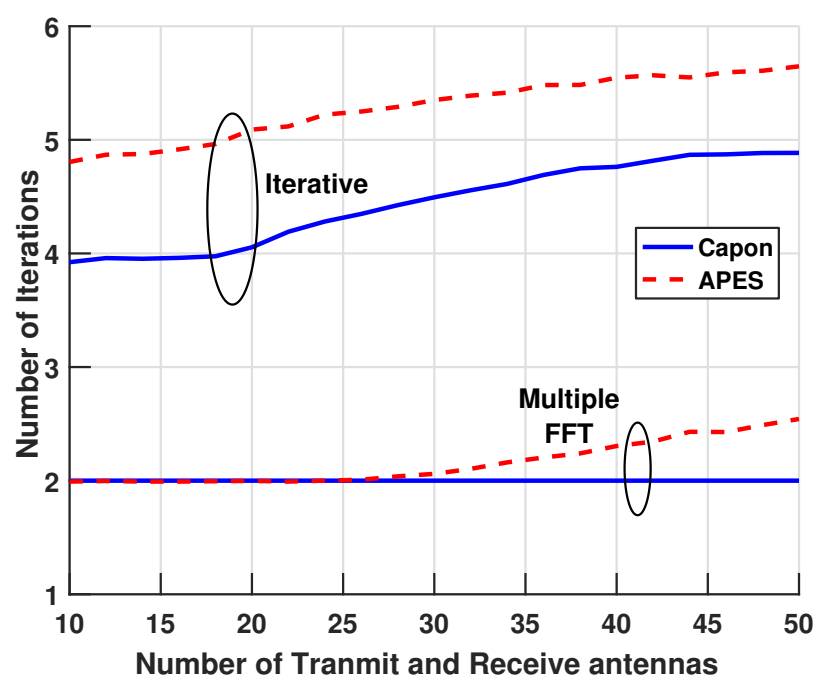

Fig. 9. Comparison of the average number of iterations needed to converge to the maximum of the Capon (solid lines) and APES (dashed lines) costfunctions using the Newton iterative algorithm or the multiple 64 2D-FFT approach under $\mathrm{SNR}=0 \mathrm{~dB}$.

\section{APPENDIX A}

The derivation of the CRLB is detailed in this section. Each entry of the vector $\frac{\partial \mathbf{u}_{n}}{\partial \boldsymbol{\eta}}$ can be expressed as follows

$$
\begin{aligned}
& \frac{\partial \mathbf{u}_{n}}{\partial \beta_{t}^{\Re}}=e^{j \omega_{t} n} \mathbf{a}_{R}\left(\bar{\omega}_{t}\right) \mathbf{a}_{T}^{T}\left(\bar{\omega}_{t}\right) \mathbf{x}_{n}=\frac{\mathbf{u}_{n}}{\beta_{t}}, \\
& \frac{\partial \mathbf{u}_{n}}{\partial \beta_{t}^{\Im}}=j e^{j \omega_{t} n} \mathbf{a}_{R}\left(\bar{\omega}_{t}\right) \mathbf{a}_{T}^{T}\left(\bar{\omega}_{t}\right) \mathbf{x}_{n}=\frac{\mathbf{u}_{n}}{-j \beta_{t}}, \\
& \frac{\partial \mathbf{u}_{n}}{\partial \omega_{t}}=j \beta_{t} n e^{j \omega_{t} n} \mathbf{a}_{R}\left(\bar{\omega}_{t}\right) \mathbf{a}_{T}^{T}\left(\bar{\omega}_{t}\right) \mathbf{x}_{n}=j n \mathbf{u}_{n}, \\
& \frac{\partial \mathbf{u}_{n}}{\partial \bar{\omega}_{t}}=j \beta_{t} e^{j \omega_{t} n} \times \\
& \left(\gamma \mathbf{a}_{T}^{T}\left(\bar{\omega}_{t}\right) \mathbf{D}_{n_{T}} \mathbf{x}_{n} \mathbf{a}_{R}\left(\bar{\omega}_{t}\right)+\mathbf{a}_{T}^{T}\left(\bar{\omega}_{t}\right) \mathbf{x}_{n} \mathbf{D}_{n_{R}} \mathbf{a}_{R}\left(\bar{\omega}_{t}\right)\right)
\end{aligned}
$$

Thus, using the following notation

$$
\begin{aligned}
& \tilde{p}_{0}\left(n, \bar{\omega}_{t}\right)=\mathbf{a}_{T}^{T}\left(\bar{\omega}_{t}\right) \mathbf{x}_{n} \mathbf{x}_{n}^{H} \mathbf{a}_{T}^{*}\left(\bar{\omega}_{t}\right), \\
& \tilde{p}_{1}\left(n, \bar{\omega}_{t}\right)=\mathbf{a}_{T}^{T}\left(\bar{\omega}_{t}\right) \mathbf{D}_{n_{T}} \mathbf{x}_{n} \mathbf{x}_{n}^{H} \mathbf{a}_{T}^{*}\left(\bar{\omega}_{t}\right), \\
& \tilde{p}_{2}\left(n, \bar{\omega}_{t}\right)=\mathbf{a}_{T}^{T}\left(\bar{\omega}_{t}\right) \mathbf{D}_{n_{T}} \mathbf{x}_{n} \mathbf{x}_{n}^{H} \mathbf{D}_{n_{T}} \mathbf{a}_{T}^{*}\left(\bar{\omega}_{t}\right),
\end{aligned}
$$

the FIM corresponding to the estimation of $\boldsymbol{\eta}$ can be represented as in (A.2). Using the following identity

$$
\begin{aligned}
q\left(\bar{\omega}_{t}\right) & =\mathbf{a}_{R}^{H}\left(\bar{\omega}_{t}\right) \mathbf{R}_{z}^{-1} \mathbf{a}_{R}\left(\bar{\omega}_{t}\right), \\
q_{1}\left(\bar{\omega}_{t}\right) & =\frac{1}{q\left(\bar{\omega}_{t}\right)} \mathbf{a}_{R}^{H}\left(\bar{\omega}_{t}\right) \mathbf{D}_{n_{R}} \mathbf{R}_{z}^{-1} \mathbf{a}_{R}\left(\bar{\omega}_{t}\right), \\
q_{2}\left(\bar{\omega}_{t}\right) & =\frac{1}{q\left(\bar{\omega}_{t}\right)} \mathbf{a}_{R}^{H}\left(\bar{\omega}_{t}\right) \mathbf{D}_{n_{R}} \mathbf{R}_{z}^{-1} \mathbf{D}_{n_{R}} \mathbf{a}_{R}\left(\bar{\omega}_{t}\right),
\end{aligned}
$$

we can express the term $f(1)$ as shown below

$$
\begin{aligned}
f(1) & =\frac{1}{q\left(\bar{\omega}_{t}\right)}\left(\frac{\mathbf{u}_{n}^{H}}{\beta_{t}^{*}} \frac{\partial \mathbf{u}_{n}}{\partial \bar{\omega}_{t}}\right)^{\Re} \\
& =\left(j \beta_{t}\left[\gamma \tilde{p}_{1}\left(n, \bar{\omega}_{t}\right)+q_{1}\left(\bar{\omega}_{t}\right) \tilde{p}_{0}\left(n, \bar{\omega}_{t}\right)\right]\right)^{\Re} \\
& =-\left(\beta_{t} q_{1}\left(\bar{\omega}_{t}\right)\right)^{\Im} \tilde{p}_{0}\left(n, \bar{\omega}_{t}\right)-\gamma\left(\beta_{t} \tilde{p}_{1}\left(n, \bar{\omega}_{t}\right)\right)^{\Im} .
\end{aligned}
$$




$$
\begin{aligned}
& \mathbf{F}=2 \mathbf{a}_{R}^{H}\left(\bar{\omega}_{t}\right) \mathbf{R}_{z}^{-1} \mathbf{a}_{R}\left(\bar{\omega}_{t}\right) \sum_{n=0}^{N-1}\left(\begin{array}{cccc}
\tilde{p}_{0}\left(n, \bar{\omega}_{t}\right) & 0 & -n \beta_{t}^{\Im} \tilde{p}_{0}\left(n, \bar{\omega}_{t}\right) & f(1) \\
0 & \tilde{p}_{0}\left(n, \bar{\omega}_{t}\right) & n \beta_{t}^{\Re} \tilde{p}_{0}\left(n, \bar{\omega}_{t}\right) & f(2) \\
-n \beta_{t}^{\Im} \tilde{p}_{0}\left(n, \bar{\omega}_{t}\right) & n \beta_{t}^{\Re} \tilde{p}_{0}\left(n, \bar{\omega}_{t}\right) & n^{2}\left|\beta_{t}\right|^{2} \tilde{p}_{0}\left(n, \bar{\omega}_{t}\right) & f(3) \\
f(1) & f(2) & f(3) & f(4)
\end{array}\right), \\
& \mathbf{F}=2 N q p\left[\begin{array}{cccc}
1 & 0 & -p_{10} \beta_{t}^{\Im} & -\left(\beta_{t}\left(q_{1}+\gamma p_{01}\right)\right)^{\Im} \\
0 & 1 & p_{10} \beta_{t}^{\Re} & \left(\beta_{t}\left(q_{1}+\gamma p_{01}\right)\right)^{\Re} \\
-p_{10} \beta_{t}^{\Im} & p_{10} \beta_{t}^{\Re} & \left|\beta_{t}\right|^{2} p_{20} & \left|\beta_{t}\right|^{2}\left(q_{1}^{\Re} p_{10}+\gamma p_{11}^{\Re}\right) \\
-\left(\beta_{t}\left(q_{1}+\gamma p_{01}\right)\right)^{\Im} & \left(\beta_{t}\left(q_{1}+\gamma p_{01}\right)\right)^{\Re} & \left|\beta_{t}\right|^{2}\left(q_{1}^{\Re} p_{10}+\gamma p_{11}^{\Re}\right) & \left|\beta_{t}\right|^{2}\left(\gamma^{2} p_{02}+2 \gamma\left(q_{1} p_{01}\right)^{\Re}+q_{2}\right)
\end{array}\right], \\
& \mathbf{M}=\left(\mathbf{B}-\mathbf{D A}^{-1} \mathbf{C}\right)^{-1}=\frac{1}{\alpha} \times\left[\begin{array}{cc}
\gamma^{2}\left(p_{02}-\left|p_{01}\right|^{2}\right)+q_{2}-\left|q_{1}\right|^{2} & \gamma\left(p_{01}^{\Re} p_{10}-p_{11}^{\Re}\right) \\
\gamma\left(p_{01}^{\Re} p_{10}-p_{11}^{\Re}\right) & p_{20}-p_{10}^{2}
\end{array}\right] \\
& \mathbf{N}=\left[\begin{array}{c}
-\mathbf{A}^{-1} \mathbf{C} \\
\mathbf{I}_{2 \times 2}
\end{array}\right]=\left[\begin{array}{cc}
p_{10} \beta_{t}^{\Im} & \left(\beta_{t}\left(q_{1}+\gamma p_{01}\right)\right)^{\Im} \\
-p_{10} \beta_{t}^{\Re} & -\left(\beta_{t}\left(q_{1}+\gamma p_{01}\right)\right)^{\Re} \\
1 & 0 \\
0 & 1
\end{array}\right]
\end{aligned}
$$

Similarly, the second term $f(2)$ can be derived as follows

$$
\begin{aligned}
f(2) & =\frac{1}{q\left(\bar{\omega}_{t}\right)}\left(\frac{\mathbf{u}_{n}^{H}}{j \beta_{t}^{*}} \frac{\partial \mathbf{u}_{n}}{\partial \bar{\omega}_{t}}\right)^{\Re} \\
& =\left(\beta_{t} q_{1}\left(\bar{\omega}_{t}\right)\right)^{\Re} \tilde{p}_{0}\left(n, \bar{\omega}_{t}\right)+\gamma\left(\beta_{t} \tilde{p}_{1}\left(n, \bar{\omega}_{t}\right)\right)^{\Re} .
\end{aligned}
$$

Next, the third term $f(3)$ is expressed as follows

$$
\begin{aligned}
f(3) & =\frac{1}{q\left(\bar{\omega}_{t}\right)}\left((-j n) \mathbf{u}_{n}^{H} \frac{\partial \mathbf{u}_{n}}{\partial \bar{\omega}_{t}}\right)^{\Re} \\
& =n\left|\beta_{t}\right|^{2}\left(q_{1}^{\Re}\left(\bar{\omega}_{t}\right) \tilde{p}_{0}\left(n, \bar{\omega}_{t}\right)+\gamma \tilde{p}_{1}^{\Re}\left(n, \bar{\omega}_{t}\right)\right) .
\end{aligned}
$$

Finally, the last term $f(4)$ is

$$
\begin{aligned}
f(4)= & \frac{1}{n_{R}}\left(\frac{\partial \mathbf{u}_{n}^{H}}{\partial \bar{\omega}_{t}} \frac{\partial \mathbf{u}_{n}}{\partial \bar{\omega}_{t}}\right)^{\Re} \\
= & \left|\beta_{t}\right|^{2}\left(\gamma^{2} \tilde{p}_{2}\left(n, \bar{\omega}_{t}\right)+2 \gamma\left(q_{1}\left(\bar{\omega}_{t}\right) \tilde{p}_{1}\left(n, \bar{\omega}_{t}\right)\right)^{\Re}\right. \\
& \left.\quad+q_{2}\left(\bar{\omega}_{t}\right) \tilde{p}_{0}\left(n, \bar{\omega}_{t}\right)\right) .
\end{aligned}
$$

The FIM can then be expressed as in (A.7) where $p_{01}, p_{02}$, $p_{10}, p_{11}$, and $p_{20}$ are defined as

$$
p_{i j}\left(\bar{\omega}_{t}\right)=\frac{\sum_{n=0}^{N-1} n^{i} \tilde{p}_{j}\left(n, \bar{\omega}_{t}\right)}{N p\left(\bar{\omega}_{t}\right)} \text {, for } i, j \in\{0,1,2\} \text {. }
$$

For notational simplicity, we did not show explicitly the dependence of $p, p_{i j}$ and $q_{i}$ on $\bar{\omega}_{t}$.

Using the matrix inversion lemma

$$
\begin{aligned}
& {\left[\begin{array}{ll}
\mathbf{A}_{2 \times 2} & \mathbf{C}_{2 \times 2} \\
\mathbf{D}_{2 \times 2} & \mathbf{B}_{2 \times 2}
\end{array}\right]^{-1}=\left[\begin{array}{l}
\mathbf{I}_{2 \times 2} \\
\mathbf{0}_{2 \times 2}
\end{array}\right] \mathbf{A}^{-1}\left[\begin{array}{ll}
\mathbf{I}_{2 \times 2} & \mathbf{0}_{2 \times 2}
\end{array}\right]} \\
& \quad+\left[\begin{array}{c}
-\mathbf{A}^{-1} \mathbf{C} \\
\mathbf{I}_{2 \times 2}
\end{array}\right]\left(\mathbf{B}-\mathbf{D A}^{-1} \mathbf{C}\right)^{-1}\left[\begin{array}{ll}
-\mathbf{D A}^{-1} & \mathbf{I}_{2 \times 2}
\end{array}\right]
\end{aligned}
$$

the CRLB of the parameters can be found as

$$
\operatorname{CRLB}(\boldsymbol{\eta})=\frac{1}{2 N q p}\left(\left[\begin{array}{ll}
\mathbf{I}_{2 \times 2} & 0_{2 \times 2} \\
0_{2 \times 2} & 0_{2 \times 2}
\end{array}\right]+\mathbf{N M N}^{T}\right),
$$

where $\mathbf{M}$ and $\mathbf{N}$ are respectively defined in (A.11) and (A.12), and

$$
\begin{aligned}
\alpha= & \left|\beta_{t}\right|^{2}\left(p_{20}-p_{10}^{2}\right)\left(q_{2}-\left|q_{1}\right|^{2}+\gamma^{2}\left(p_{02}-\left|p_{01}\right|^{2}\right)\right) \\
& -\left|\beta_{t}\right|^{2} \gamma^{2}\left(p_{11}^{\Re}-p_{01}^{\Re} p_{10}\right)^{2} .
\end{aligned}
$$

Therefore, the final expressions of the CLRB for the different parameters are detailed below

$$
\begin{aligned}
& \operatorname{CRLB}\left(\beta_{t}^{\Re}\right)=\frac{1}{2 N q p}\left(1+\frac{\Delta_{1} \beta_{t}^{\Im^{2}}+\Delta_{2} \beta_{t}^{\Im} \beta_{t}^{\Re}+\Delta_{3} \beta_{t}^{\Re^{2}}}{\alpha}\right), \\
& \operatorname{CRLB}\left(\beta_{t}^{\Im}\right)=\frac{1}{2 N q p}\left(1+\frac{\Delta_{1} \beta_{t}^{\Re^{2}}-\Delta_{2} \beta_{t}^{\Im} \beta_{t}^{\Re}+\Delta_{3} \beta_{t}^{\Im^{2}}}{\alpha}\right), \\
& \operatorname{CRLB}\left(\omega_{t}\right)=\frac{1}{2 N q p} \frac{q_{2}-\left|q_{1}\right|^{2}+\gamma^{2}\left(p_{02}-\left|p_{01}\right|^{2}\right)}{\alpha}, \\
& \operatorname{CRLB}\left(\bar{\omega}_{t}\right)=\frac{1}{2 N q p} \frac{p_{20}-p_{10}^{2}}{\alpha},
\end{aligned}
$$

where

$$
\begin{aligned}
\Delta_{1}= & p_{10}^{2}\left(q_{2}-\left|q_{1}\right|^{2}+\gamma^{2}\left(p_{02}-\left|p_{01}\right|^{2}\right)\right) \\
& +2 \gamma p_{10}\left(p_{10} p_{01}^{\Re}-p_{11}^{\Re}\right)\left(q_{1}^{\Re}+\gamma p_{01}^{\Re}\right) \\
& +\left(p_{20}-p_{10}^{2}\right)\left(q_{1}^{\Re}+\gamma p_{01}^{\Re}\right)^{2}, \\
\Delta_{2}= & 2\left(q_{1}^{\Im}+\gamma p_{01}^{\Im}\right)\left(\gamma p_{20} p_{01}^{\Re}-\gamma p_{10} p_{11}^{\Re}+q_{1}^{\Re}\left(p_{20}-p_{10}^{2}\right)\right), \\
\Delta_{3}= & \left(p_{20}-p_{10}^{2}\right)\left(q_{1}^{\Im}+\gamma p_{01}^{\Im}\right)^{2} .
\end{aligned}
$$

\section{APPENDIX B}

To implement the Newton algorithm, the first and second order derivatives with respect to $\omega$ and $\bar{\omega}$ of the following three expressions

$$
\begin{aligned}
|\mathcal{S}(\omega, \bar{\omega})|^{2} & =\left|E\left\{e^{-j \omega n} \mathbf{a}_{R}^{H}(\bar{\omega}) \mathbf{r}_{n} \mathbf{x}_{n}^{H} \mathbf{a}_{T}^{*}(\bar{\omega})\right\}\right|^{2}, \\
\|\mathcal{V}(\omega, \bar{\omega})\|_{2}^{2} & =\sum_{i=1}^{n_{R}}\left|E\left\{e^{-j \omega n} \tilde{r}_{i, n} \mathbf{x}_{n}^{H} \mathbf{a}_{T}^{*}(\bar{\omega})\right\}\right|^{2}
\end{aligned}
$$

and

$$
f(\bar{\omega})=\mathbf{a}_{R}^{H}(\bar{\omega}) \mathbf{R}_{y}^{-1} \mathbf{a}_{R}(\bar{\omega})
$$


are required.

Using matrix transformation, (B.1) can be reformulated as

$$
|\mathcal{S}(\omega, \bar{\omega})|^{2}=\left|\frac{1}{N} \sum_{n=0}^{N-1} e^{-j \omega n} \mathbf{x}_{n}^{H} \tilde{\mathbf{R}}_{n}^{T} \mathbf{a}^{*}(\bar{\omega})\right|^{2},
$$

where $\tilde{\mathbf{R}}_{n}$ is defined in (11) and

$$
\mathbf{a}(\bar{\omega})=\left[\begin{array}{llll}
1 & e^{j \bar{\omega}} & \cdots & e^{j \bar{\omega}\left(\gamma\left(n_{T}-1\right)+n_{R}-1\right)}
\end{array}\right]^{T} .
$$

The derivatives of $|\mathcal{S}(\omega, \bar{\omega})|^{2}$ with respect to $\omega$ and $\bar{\omega}$ are respectively computed below

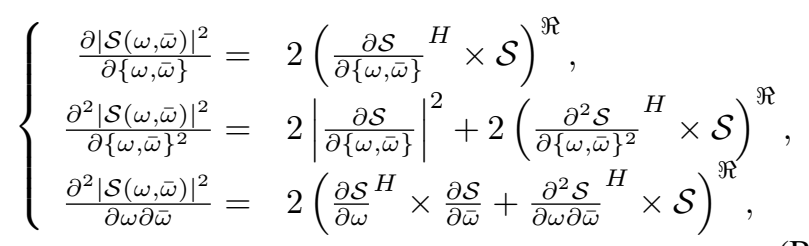

where

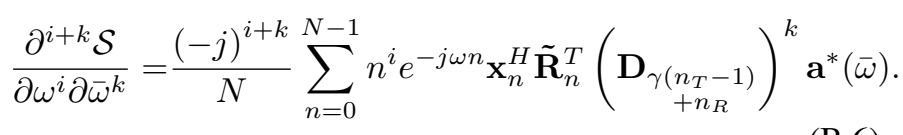

Next, the derivatives of $\|\mathcal{V}(\omega, \bar{\omega})\|_{2}^{2}$ will be similar to (B.5) but expressed as function of

$$
\frac{\partial^{i+k} \mathcal{V}}{\partial \omega^{i} \partial \bar{\omega}^{k}}=\frac{(-j)^{i+k} \gamma^{k}}{N} \sum_{n=0}^{N-1} n^{i} e^{-j \omega n} \tilde{\mathbf{r}}_{n} \mathbf{x}_{n}^{H}\left(\mathbf{D}_{n_{T}}\right)^{k} \mathbf{a}_{T}^{*}(\bar{\omega}) .
$$

It should be noted that the entities $\mathbf{x}_{n}^{H} \tilde{\mathbf{R}}_{n}^{T}$ and $\tilde{\mathbf{r}}_{n} \mathbf{x}_{n}^{H}$ are independent of $\omega$ and $\bar{\omega}$, thus, do not vary from one iteration to another. Therefore, to reduce the computational complexity of the iterative algorithm, these entities are evaluated only once for all time samples $n$.

Considering the symmetry of $\mathbf{R}_{y}^{-1}$, the third expression $f(\bar{\omega})$ can be reformulated as follows

$$
\begin{aligned}
f(\bar{\omega}) & =\sum_{p=0}^{n_{R}-1} \sum_{q=0}^{n_{R}-1}\left(\mathbf{R}_{y}^{-1}\right)_{p, q} e^{j \bar{\omega}(q-p)} \\
& =\sum_{p=0}^{n_{R}-1}\left(\mathbf{R}_{y}^{-1}\right)_{p, p}+2\left(\sum_{p=0}^{n_{R}-2} \sum_{q>p}^{n_{R}-1}\left(\mathbf{R}_{y}^{-1}\right)_{p, q} e^{j \bar{\omega}(q-p)}\right)^{\Re} \\
& =2\left(\mathbf{f}^{T} \mathbf{a}_{R}(\bar{\omega})\right)^{\Re}
\end{aligned}
$$

where

$$
\mathbf{f}=\left[\begin{array}{llll}
\frac{1}{2} \sum_{p=0}^{n_{R}-1}\left(\mathbf{R}_{y}^{-1}\right)_{p, p} & \sum_{p=0}^{n_{R}-2}\left(\mathbf{R}_{y}^{-1}\right)_{p, p+1} & \cdots & \left(\mathbf{R}_{y}^{-1}\right)_{0, n_{R}-1}
\end{array}\right]^{T}
$$

Consequently, the derivative of $f$ with respect to $\bar{\omega}$ can be expressed as below

$$
\frac{\partial^{i} f(\bar{\omega})}{\partial \bar{\omega}^{i}}=2\left(\mathbf{f}^{T}\left(j \mathbf{D}_{n_{R}}\right)^{i} \mathbf{a}_{R}(\bar{\omega})\right)^{\Re} .
$$

Similarly, the reader can easily verify that

$$
\frac{\partial^{i} p(\bar{\omega})}{\partial \bar{\omega}^{i}}=\frac{\partial^{i}}{\partial \bar{\omega}^{i}}\left(\mathbf{a}_{T}^{T}(\bar{\omega}) \mathbf{R}_{x} \mathbf{a}_{T}^{*}(\bar{\omega})\right)
$$

$$
=2\left(\mathbf{r}_{x}^{T}\left(-j \gamma \mathbf{D}_{n_{T}}\right)^{i} \mathbf{a}_{T}^{*}(\bar{\omega})\right)^{\Re},
$$

where

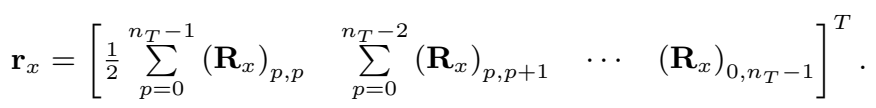

Using the first and second order derivatives of $|\mathcal{S}(\omega, \bar{\omega})|^{2}$, $\|\mathcal{V}(\omega, \bar{\omega})\|_{2}^{2}, f(\bar{\omega})$ and $p(\bar{\omega})$, the derivative of the cost functions (12) and, (24) defined in Section III can be easily deduced.

\section{REFERENCES}

[1] J. Li and P. Stoica, "MIMO radar with colocated antennas," IEEE Signal Processing Magazine, vol. 24, pp. 106-114, Sept. 2007.

[2] A. M. Haimovich, R. S. Blum, and L. J. Cimini, "MIMO radar with widely separated antennas," IEEE Signal Processing Magazine, vol. 25, pp. 116-129, Jan. 2008.

[3] E. Fishler, A. Haimovich, R. Blum, D. Chizhik, L. Cimini, and R. Valenzuela, "MIMO radar: an idea whose time has come," in Proceedings of the IEEE Radar Conference, pp. 71-78, Apr. 2004.

[4] E. Fishler, A. Haimovich, R. Blum, L. Cimini, D. Chizhik, and R. Valenzuela, "Spatial diversity in radars-models and detection performance," IEEE Transactions on Signal Processing, vol. 54, pp. 823-838, Mar 2006.

[5] J. Li, P. Stoica, L. Xu, and W. Roberts, "On parameter identifiability of MIMO radar," IEEE Signal Processing Letters, vol. 14, pp. 968-971, Dec. 2007.

[6] S. Ahmed, J. S. Thompson, and B. Mulgrew, "Unconstrained synthesis of covariance matrix for MIMO radar transmit beampattern," IEEE Transactions on Signal Processing, vol. 59, pp. 3837 - 3849, Aug. 2011.

[7] S. Jardak, S. Ahmed, and M. Alouini, "Generation of correlated finite alphabet waveforms using Gaussian random variables," IEEE Transactions on Signal Processing, vol. 62, no. 17, pp. 4587-4596, 2014.

[8] E. Fishler, A. Haimovich, R. Blum, R. Cimini, D. Chizhik, and R. Valenzuela, "Performance of MIMO radar systems: advantages of angular diversity," in Conference Record of the Thirty-Eighth Asilomar Conference on Signals, Systems and Computers, vol. 1, pp. 305-309, Nov. 2004.

[9] J. Li and P. Stoica, MIMO Radar Signal Processing - Diversity Means Superiority. Hoboken New Jersey: John Wiley \& Sons, Inc., 2008.

[10] L. Xu, J. Li, and P. Stoica, "Target detection and parameter estimation for MIMO radar systems," IEEE Transactions on Aerospace and Electronic Systems, vol. 44, pp. 927-939, July 2008.

[11] H. Yan, J. Li, and G. Liao, "Multitarget identification and localization using bistatic MIMO radar systems," EURASIP Journal on Advanced Signal Processing, vol. 2008, pp. 48:1-48:8, Jan. 2008.

[12] D. Nion and N. D. Sidiropoulos, "A PARAFAC-based technique for detection and localization of multiple targets in a MIMO radar system," in IEEE International Conference on Acoustics, Speech and Signal Processing, pp. 2077-2080, April 2009.

[13] C. Yunhe, "Joint estimation of angle and Doppler frequency for bistatic MIMO radar," Electronics Letters, vol. 46, pp. 170-172, Jan. 2010.

[14] K. Rambach and B. Yang, "Direction of Arrival estimation of two moving targets using a time division multiplexed colocated MIMO radar," in IEEE Radar Conference, pp. 1118-1123, May 2014.

[15] K. Rambach and B. Yang, "Colocated MIMO radar: Cramer-Rao bound and optimal time division multiplexing for DOA estimation of moving targets," in IEEE International Conference on Acoustics, Speech and Signal Processing (ICASSP), pp. 4006-4010, May 2013.

[16] S. R. Alty, A. Jakobsson, and E. G. Larsson, "Efficient implementation of the time-recursive Capon and APES spectral estimators," in 12th European Signal Processing Conference, pp. 1269-1272, Sept. 2004.

[17] T. Ekman, A. Jakobsson, and P. Stoica, "On efficient implementation of the capon algorithm," in European Association Signal Processing Conference, Tampere, Finland, Sept. 2000.

[18] E. Larsson and P. Stoica, "Fast implementation of two-dimensional APES and CAPON spectral estimators," Multidimensional Systems and Signal Processing, vol. 13, no. 1, pp. 35-53, 2002.

[19] E. Larsson and P. Stoica, "Fast implementation of two-dimensional APES and CAPON spectral estimators," in IEEE International Conference on Acoustics, Speech, and Signal Processing, vol. 5, pp. 3069-3072 vol.5, 2001. 
[20] J. Li and P. Stoica, "An adaptive filtering approach to spectral estimation and SAR imaging," IEEE Transactions on Signal Processing, vol. 44, pp. 1469-1484, June 1996.

[21] Z. S. Liu, H. Li, and J. Li, "Efficient implementation of Capon and APES for spectral estimation," IEEE Transactions on Aerospace and Electronic Systems, vol. 34, pp. 1314-1319, Oct. 1998.

[22] J. Capon, "High-resolution frequency-wavenumber spectrum analysis," Proceedings of the IEEE, vol. 57, no. 8, pp. 1408-1418, 1969.

[23] H. Krim and M. Viberg, "Two decades of array signal processing research: the parametric approach,” IEEE Signal Processing Magazine, vol. 13, pp. 67-94, July 1996.

[24] P. Stoica, H. Li, and J. Li, "A new derivation of the APES filter," IEEE Signal Processing Letters, vol. 6, pp. 205-206, Aug. 1999.

[25] C. Richmond, "On the probability of resolution for the amplitude and phase estimation (APES) spectral estimator," in IEEE International Conference on Acoustics, Speech, and Signal Processing, vol. 4, pp. 10251028, Mar. 2005.

[26] G. H. Golub and C. F. Van Loan, Matrix Computations (3rd Ed.). Baltimore, MD, USA: Johns Hopkins University Press, 1996.

[27] P. Stoica and R. Moses, Introduction to Spectral Analysis. Prentice Hall, 1997.

[28] S. Jardak, S. Ahmed, and M.-S. Alouini, "Low complexity joint estimation of reflection coefficient, spatial location, and Doppler shift for MIMO radar by exploiting 2D-FFT," International Radar Conference, Lille, France, Oct. 2014.

[29] D. Bliss and K. Forsythe, "Multiple-input multiple-output (MIMO) radar and imaging: degrees of freedom and resolution," in Conference Record of the Thirty-Seventh Asilomar Conference on Signals, Systems and Computers, 2004., vol. 1, pp. 54-59, Nov. 2003.

[30] K. Forsythe, D. Bliss, and G. S. Fawcett, "Multiple-input multiple-output (MIMO) radar: performance issues," in Conference Record of the ThirtyEighth Asilomar Conference on Signals, Systems and Computers, vol. 1, pp. 310-315, Nov. 2004.

[31] D. J. Rabideau and P. Parker, "Ubiquitous MIMO multifunction digital array radar," in Conference Record of the Thirty-Seventh Asilomar Conference on Signals, Systems and Computers, 2004., vol. 1, pp. 10571064, Nov. 2003.

[32] F. Robey, S. Coutts, D. Weikle, J. McHarg, and K. Cuomo, "MIMO radar theory and experimental results," in Conference Record of the ThirtyEighth Asilomar Conference on Signals, Systems and Computers, vol. 1, pp. 300-304, Nov. 2004.

[33] S. Boyd and L. Vandenberghe, Convex Optimization. New York, NY, USA: Cambridge University Press, 2004.

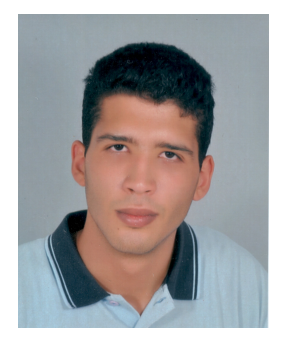

Seifallah Jardak received the Diplôme d'Ingénieur degree from the École Polytechnique de Tunisie (EPT), La Marsa, Tunisia, in 2012. Afterwards, he continued his Master studies in King Abdullah University of Science and Technology (KAUST) and received his Master of Science degree in Electrical Engineering in April 2014. He is currently a Ph.D student at KAUST. His research interests lie mainly in the field of MIMO and passive radar, with current research focusing on the design and implementation of a portable low-cost FMCW radar.

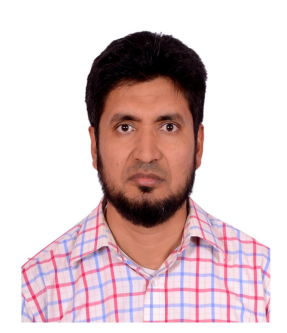

Sajid Ahmed (M'08, SM'12) received the B.S in Electronics Engineering from Sir Syed University of Engiering and Technology Karachi, Pakistan in 1998 and M. Sc in Communication Engineering from University of Manchester, Institute of Science and Technology, UK in 2002. He completed his PhD in Digital Signal Processing at the King's College London and Cardiff University, UK in 2005. He was a researcher at the Queen's University Belfast, Northern Ireland and the University of Edinburgh, UK. Presently, he is with the King Abdullah University of Science and Technology (KAUST), Thuwal, Kingdom of Saudi Arabia. Dr. Ahmed's current research interests include the linear and nonlinear optimisation techniques, low complexity parameter estimation for communication and radar systems, passive radar, and waveforms design for MIMO radar.

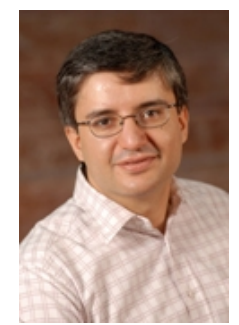

Mohamed-Slim Alouini (S'94, M'98, SM'03, F09) was born in Tunis, Tunisia. He received the Ph.D. degree in Electrical Engineering from the California Institute of Technology (Caltech), Pasadena, CA, USA, in 1998. He served as a faculty member in the University of Minnesota, Minneapolis, MN, USA, then in the Texas A\&M University at Qatar, Education City, Doha, Qatar before joining King Abdullah University of Science and Technology (KAUST), Thuwal, Makkah Province, Saudi Arabia as a Professor of Electrical Engineering in 2009. His current research interests include the modeling, design, and performance analysis of wireless communication systems. 